\section{Case Reports in Oncology}

Case Rep Oncol 2019;12:548-553

DOI: $10.1159 / 000501715$

Published online: July 16, 2019

(C) 2019 The Author(s)

Published by S. Karger AG, Base

www.karger.com/cro

This article is licensed under the Creative Commons Attribution-NonCommercial 4.0 International License (CC BY-NC) (http://www.karger.com/Services/OpenAccessLicense).

Usage and distribution for commercial purposes requires written permission.

\title{
Successful Resection of Cisplatin- Resistant Renal Pelvic Cancer after the Administration of Pembrolizumab as Second-Line Therapy
}

\author{
Takashi Kawahara $^{a}$ Taku Mochizuki ${ }^{a}$ Rumiko Sugimura ${ }^{a}$ Koji Izumia \\ Shinnosuke Kuroda ${ }^{a}$ Yasuhide Miyoshi ${ }^{a}$ Noboru Nakaigawa ${ }^{b}$ \\ Masahiro Yao $^{b}$ Mikiko Tanabe $^{c}$ Hiroji Uemura $^{a}$ \\ aDepartments of Urology and Renal Transplantation, Yokohama City University Medical \\ Center, Yokohama, Japan; 'bepartment of Urology, Yokohama City University Graduate \\ School of Medicine, Yokohama, Japan; 'Division of Diagnostic pathology, Yokohama City \\ University Medical Center, Yokohama, Japan
}

\section{Keywords}

Pembrolizumab · Renal pelvic cancer · Urothelial carcinoma - Neo-adjuvant

\begin{abstract}
Pembrolizumab has been used as a second-line systemic therapy for urothelial carcinoma. We herein report a case of cisplatin-resistant renal-pelvic urothelial carcinoma that was successfully resected after pembrolizumab treatment. A 74-year-old woman was referred to our hospital for further examination for gross hematuria and a renal-pelvis tumor. Retrograde pyelography showed a defect lesion in her renal pelvis and urinary cytology of the renal pelvis showed class V. Because staging CT could not deny lung metastasis, we planned to perform nephroureterectomy after evaluating the response to neoadjuvant chemotherapy. After three courses of gemcitabine and cisplatin chemotherapy, the original site showed progression; thus, nephro-ureterectomy was cancelled. We introduced pembrolizumab as a second-line therapy. After four courses of pembrolizumab treatment, the size of the original lesion was significantly decreased. During these therapies the lung tumor size was unchanged; thus, we determined that the lung tumor was not metastatic and performed nephro-ureterectomy. A pathological
\end{abstract}




\section{Case Reports in Oncology}

examination demonstrated that the tumor was completely resected with a negative surgical margin. We described the first case in which cisplatin-resistant renal pelvic tumor was successfully resected after pembrolizumab treatment.

\section{Introduction}

Upper urinary tract urothelial carcinoma (UUT-UC) is sometimes diagnosed in an advanced stage and sometimes gives up complete surgical resection [1-3]. Even in cases where nephro-ureterectomy was performed, recurrence has been reported. At the time of initially metastatic or recurrence cases, cisplatin-based chemotherapy is introduced, but almost all cases develop a cisplatin-resistant status [4]. Recently, an immune checkpoint inhibitor, pembrolizumab, has been introduced as a second-line chemotherapy for cisplatin-resistant cancer. In a KEYNOTE study, pembrolizumab showed efficacy in urothelial carcinoma, and a subgroup analysis showed more favorable effect in renal pelvis cancer [5, 6]. Despite this effectiveness, a case of complete surgical resection after pembrolizumab treatment has not been reported. We herein report the first case in which cisplatin-resistant renal pelvic cancer was successfully resected after the administration of pembrolizumab chemotherapy.

\section{Case Presentation}

A 74-year-old woman was referred to our hospital due to gross hematuria in March 2018. Cystoscopy showed no bladder tumor and urinary cytology showed class V. CT revealed a right renal pelvic tumor of $6.8 \mathrm{~cm}$ diameter and a small lung tumor $(0.8 \mathrm{~cm})$ was observed (Fig. 1a, b). Retrograde pyelography showed a right renal pelvic mass and urinary cytology of her renal pelvic urine showed class V (Fig. 1c). Based on these findings, right renal pelvic cancer with suspected lung metastasis was diagnosed.

Although lung metastasis was suspected, because the tumor was small and located at a single site, we planned to perform radical nephro-ureterectomy after neoadjuvant systemic chemotherapy of gemcitabine and cisplatin (GC). After three courses of GC treatment, CT revealed progression of the mass and adherence to the intestine, with no change in the lung mass. Surgical resection was cancelled due to progression of the tumor. Pembrolizumab was administered as a second-line treatment. After four cycles of pembrolizumab treatment, the tumor decreased in size and surgical resection became possible (Fig. 2). We paused pembrolizumab treatment at the fourth cycle and planned to perform nephro-ureterectomy. The lung tumor did not change during treatment with GC or pembrolizumab. The primary tumor site continued to shrink until just before nephro-ureterectomy. During surgery the tumor was found to have severely adhered to the peritoneum. Consequently, the kidney was resected with the peritoneum (Fig. 3a, b). The final pathological diagnosis was invasive urothelial carcinoma (pT3), high grade, with a negative surgical margin (Fig. 3c, d). Most of the tumor was replaced by foamy histiocytes (Fig. $4 \mathrm{a}-\mathrm{c}$ ). The patient was free from recurrence at 6 months after surgery. 


\section{Discussion}

UUT-UC accounts for approximately $5 \%$ of urothelial cancers $[3,6,7]$. Radical nephroureterectomy is the gold standard treatment for localized UUT-UC. However, UUT-UC is correlated with a high incidence of tumor recurrence and poor patient outcomes. GC therapy is a standard treatment for advanced or recurrent UUT-UC. Based on the results of the KEYNOTE study, pembrolizumab was introduced as a second-line treatment for urothelial carcinoma in Japan. Although this showed effectiveness, there have been no previous reports showing complete surgical resection after pembrolizumab treatment $[5,6]$.

We firstly report the complete surgical resection after pembrolizumab treatment. In the KEYNOTE study, UUT-UC showed a favorable response to pembrolizumab treatment and a sub-group analysis revealed that the outcomes in cases involving renal pelvic tumors were more favorable than those involving bladder cancer [5]. We also investigated the PD-L1 expression and found that it was not expressed at the tumor site. Because no biopsy was performed before the initiation of pembrolizumab therapy, the PD-L1 expression was not evaluated before the start of pembrolizumab treatment. In non-small cell lung cancer, pembrolizumab shows significantly higher efficacy in the treatment of PD-L1-positive patients than in the treatment of PD-L1-negative patients [8]. We hypothesized that a location within the renal pelvis might be a clue to identifying cases in which pembrolizumab can be expected to show higher efficacy.

This patient underwent nephro-ureterectomy after four courses of pembrolizumab treatment because CT showed a significant decrease in the size of the tumor and we determined that surgical resection was feasible. Although a pathological examination confirmed that the tumor had diminished, it was tightly attached to the peritoneum. Thus, more courses of pembrolizumab might have contributed to easier surgical resection.

We reported a first case in which surgical resection of cisplatin-resistant tumor in the renal pelvis became possible after pembrolizumab treatment.

\section{Availability of Data and Material}

Due to ethical restrictions, the raw data underlying this paper are available upon request to the corresponding author.

\section{Statement of Ethics}

Written informed consent was obtained from the patient. A copy of the written consent form is available for review from the Editor-in-Chief of this journal. The present study received ethical approval.

\section{Disclosure Statement}

The authors declare no conflicts of interest in association with the present study. 


\section{Case Reports in Oncology}

\section{Funding Sources}

Grants from KAKENHI grants (16K20152) from the Ministry of Education, Culture, Sports, Science and Technology of Japan and a grant for the 2016-2017 Research Development Fund (No. WJ2810) of Yokohama City University.

\section{References}

1 Ohtaka M, Kawahara T, Ishiguro Y, Sharma M, Yao M, Miyamoto H, et al. Expression of receptor activator of nuclear factor kappa B ligand in bladder cancer. Int J Urol. 2018 Oct;25(10):901-2.

2 Yasui M, Kawahara T, Takamoto D, Izumi K, Uemura H, Miyamoto H. Distribution of androgen receptor expression in the urinary bladder. Int J Urol. 2019 Feb;26(2):305-6.

3 Munoz JJ, Ellison LM. Upper tract urothelial neoplasms: incidence and survival during the last 2 decades. J Urol. 2000 Nov;164(5):1523-5.

4 Kashiwagi E, Inoue S, Mizushima T, Chen J, Ide H, Kawahara T, et al. Prostaglandin receptors induce urothelial tumourigenesis as well as bladder cancer progression and cisplatin resistance presumably via modulating PTEN expression. Br J Cancer. 2018 Jan;118(2):213-23.

5 Bellmunt J, de Wit R, Vaughn DJ, Fradet Y, Lee JL, Fong L, et al.; KEYNOTE-045 Investigators. Pembrolizumab as Second-Line Therapy for Advanced Urothelial Carcinoma. N Engl J Med. 2017 Mar;376(11):1015-26.

6 Vaughn DJ, Bellmunt J, Fradet Y, Lee JL, Fong L, Vogelzang NJ, et al. Health-Related Quality-of-Life Analysis From KEYNOTE-045: A Phase III Study of Pembrolizumab Versus Chemotherapy for Previously Treated Advanced Urothelial Cancer. J Clin Oncol. 2018 Jun;36(16):1579-87.

7 Siegel R, Ma J, Zou Z, Jemal A. Cancer statistics, 2014. CA Cancer J Clin. 2014 Jan-Feb;64(1):9-29.

8 Herbst RS, Baas P, Kim DW, Felip E, Pérez-Gracia JL, Han JY, et al. Pembrolizumab versus docetaxel for previously treated, PD-L1-positive, advanced non-small-cell lung cancer (KEYNOTE-010): a randomised controlled trial. Lancet. 2016 Apr;387(10027):1540-50.
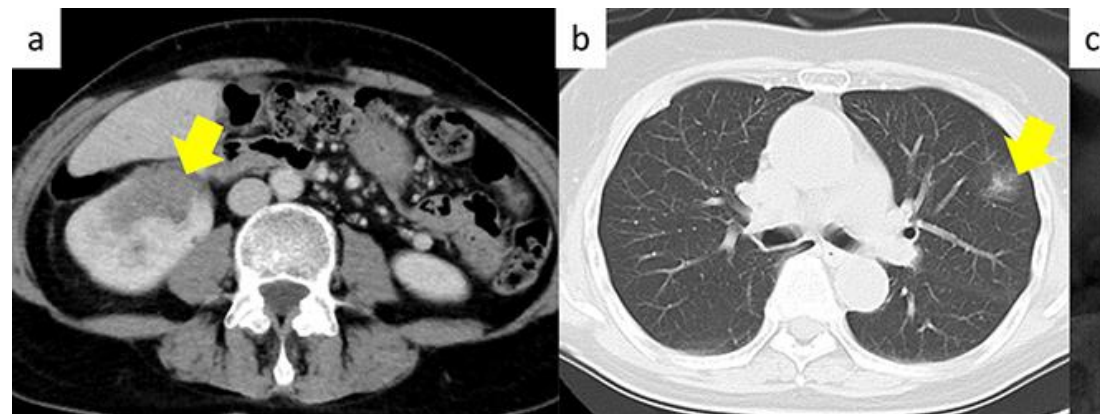

C

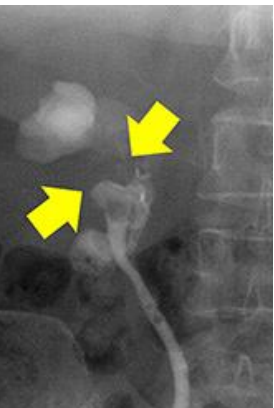

Fig. 1. a, b CT of the renal pelvic tumor and lung tumor. c Retrograde pyelography. 


\section{Case Reports in Oncology}

\begin{tabular}{l|l}
\hline Case Rep Oncol 2019;12:548-553 \\
\hline DOI: 10.1159/000501715 & $\begin{array}{l}\text { (c) 2019 The Author(s). Published by S. Karger AG, Basel } \\
\text { www.karger.com/cro }\end{array}$ \\
\hline
\end{tabular}

Kawahara et al:: Successful Resection of Cisplatin-Resistant Renal Pelvic Cancer after the Administration of Pembrolizumab as Second-Line Therapy

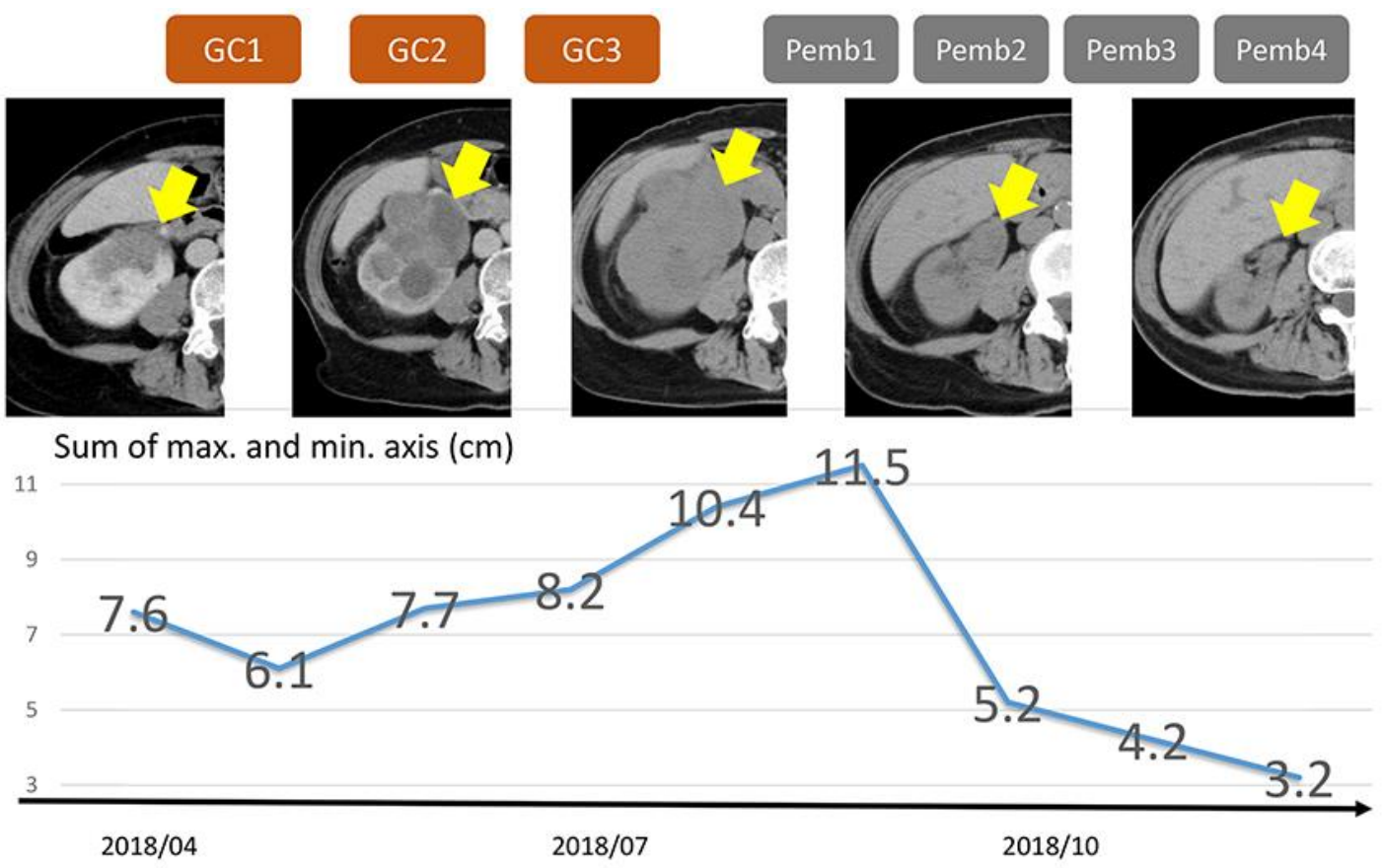

Fig. 2. The clinical course from the initial diagnosis to the pre-surgical status.
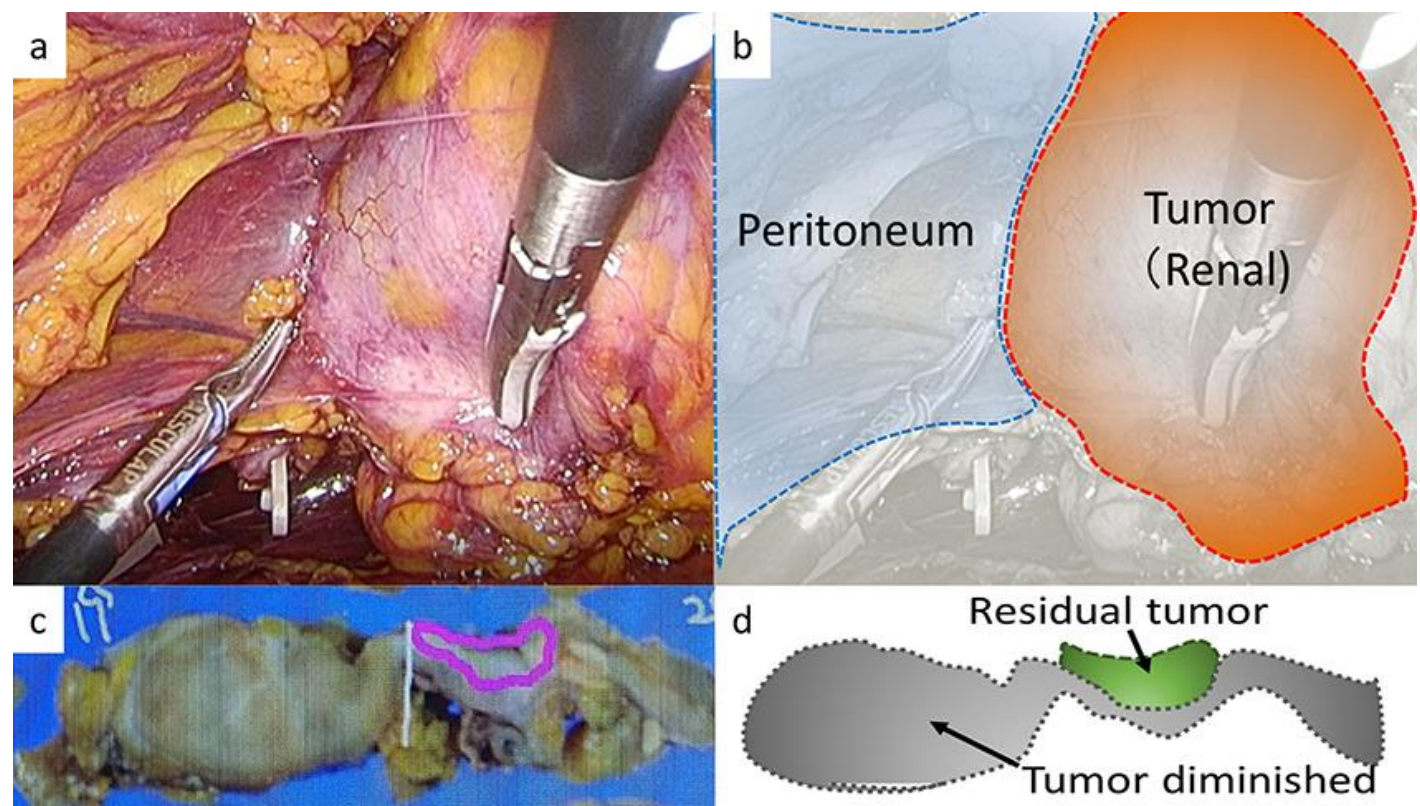

Fig. 3. a, b The tumor was tightly attached to the peritoneum and was resected with the peritoneum. c, d Most of the tumor was diminished; however, a residual tumor was still present. 


\section{Case Reports in Oncology}

Case Rep Oncol 2019;12:548-553

DOI: $10.1159 / 000501715$

(c) www.karger.com/cro

Kawahara et al:: Successful Resection of Cisplatin-Resistant Renal Pelvic Cancer after the Administration of Pembrolizumab as Second-Line Therapy
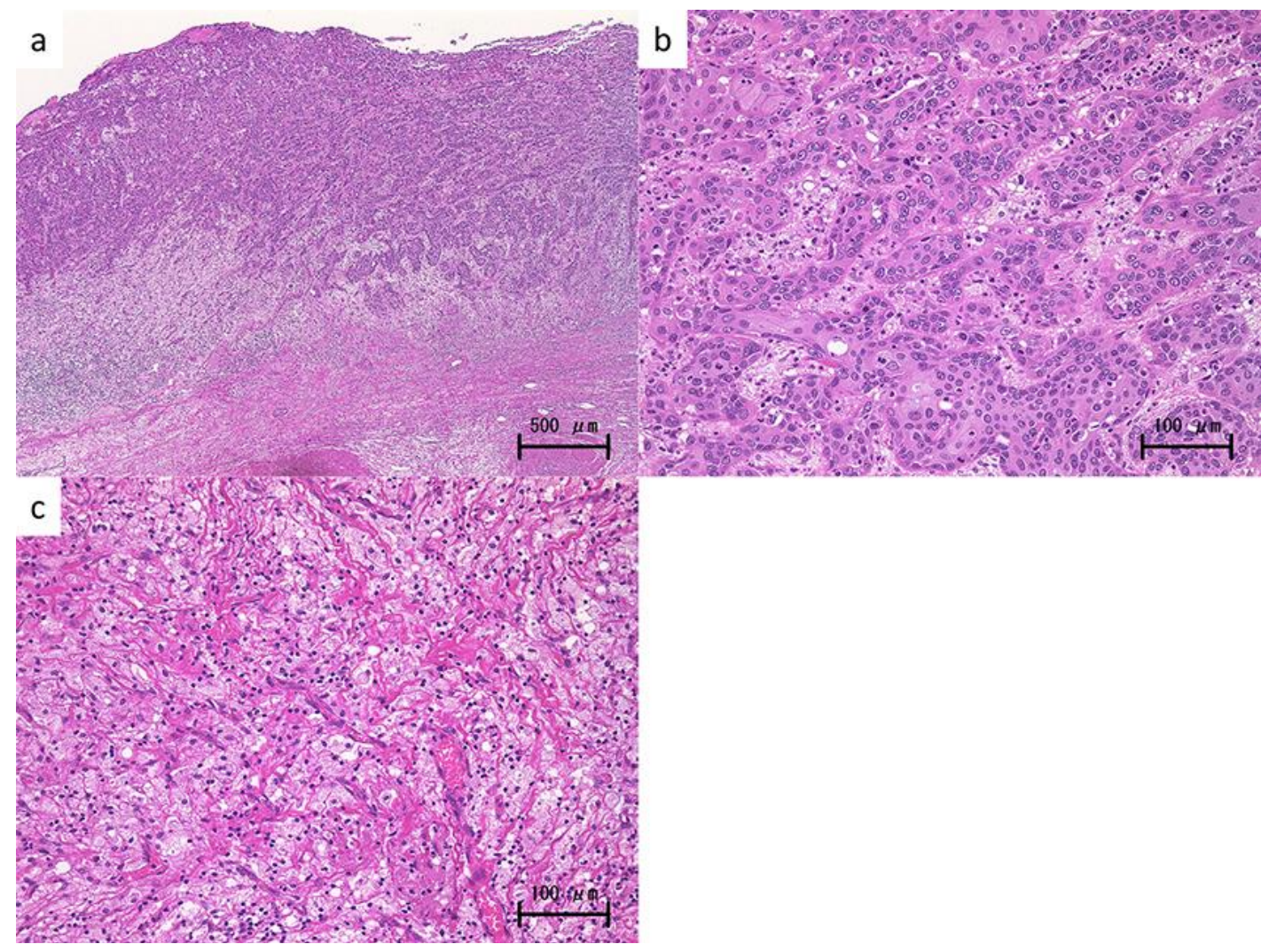

Fig. 4. a-c Hematoxylin-eosin staining of the tumor. c Most of the tumor changed to foamy histiocytes. 